\title{
Ectopic Expressions of the GhLETM1 Gene Reveal Sensitive Dose Effects on Precise Stamen Development and Male Fertility in Cotton
}

\author{
Li Zhang ${ }^{1,+}$, Yao Zhang ${ }^{1,+}$, Yijie Fan ${ }^{1}$, Haixia Guo ${ }^{1}$, Huihui Guo ${ }^{1}$, Jianfei Wu ${ }^{1}$, \\ Hongmei Wang ${ }^{2}$, Yunlei Zhao ${ }^{2}$, Xin Lian ${ }^{1}$, Zhongyuan Gou ${ }^{1}$, Yuxiao Sun ${ }^{1}$, Congcong Zheng ${ }^{1}$, \\ Cuixia Chen ${ }^{1}$ and Fanchang Zeng ${ }^{1, *(\mathbb{D})}$ \\ 1 State Key Laboratory of Crop Biology, College of Agronomy, Shandong Agricultural University, \\ Tai'an 271018, China; 15610418001@163.com (L.Z.); yaozhang188@163.com (Y.Z.); yjfan@sdau.edu.cn (Y.F.); \\ diya_haixiaguo@163.com (H.G.); hhguo@sdau.edu.cn (H.G.); jfwu@sdau.edu.cn (J.W.); \\ xlian017@163.com (X.L.); g823125157@163.com (Z.G.); 13785817706@163.com (Y.S.); zccylxq@163.com (C.Z.); \\ cxchen@sdau.edu.cn (C.C.) \\ 2 State Key Laboratory of Cotton Biology, Cotton Research Institute, Chinese Academy of Agricultural \\ Sciences, Anyang 455000, China; aywhm@163.com (H.W.); zhaoyunlei@caas.cn (Y.Z.) \\ * Correspondence: fczeng@sdau.edu.cn; Tel.: +86-538-824-1828 \\ + These authors share first authorship.
}

Received: 16 June 2020; Accepted: 7 July 2020; Published: 9 July 2020

\begin{abstract}
The homologous leucine zipper/EF-hand-containing transmembranes (LETMs) are highly conserved across a broad range of eukaryotic organisms. The LETM functional characteristics involved in biological process have been identified primarily in animals, but little is known about the LETM biological function mode in plants. Based on the results of the current investigation, the GhLETM1 gene crucially affects filament elongation and anther dehiscence of the stamen in cotton. Both excessive and lower expression of the GhLETM1 gene lead to defective stamen development, resulting in shortened filaments and indehiscent anthers with pollen abortion. The results also showed that the phenotype of the shortened filaments was negatively correlated with anther defects in the seesaw model under the ectopic expression of GhLETM1. Moreover, our results notably indicated that the gene requires accurate expression and exhibits a sensitive dose effect for its proper function. This report has important fundamental and practical significance in crop science, and has crucial prospects for genetic engineering of new cytoplasmic male sterility lines and breeding of crop hybrid varieties.
\end{abstract}

Keywords: cotton biotechnology; male sterility; shortened filaments; indehiscent anthers; pollen abortion; gene expression regulation

\section{Introduction}

Using cytoplasmic male sterile lines to cultivate hybrid varieties in crops can produce great economic and social benefits. Therefore, it is of great theoretical and practical significant to mine new genes related to fertility and develop male sterility germplasm innovation in plants. Based on our preliminary mutant analysis earlier, we discovered that the leucine zipper/EF-hand-containing transmembrane (LETM) gene was associated with mutant phenotype of male sterility. LETM encodes the protein of the inner mitochondrial membrane. LETM contains only one transmembrane helix, but it behaves as a putative transporter. The homologous LETMs have conserved sequences in lower eukaryotes, animals and plants [1]. In animals, studies have shown that LETM mediated the regulation of mitochondrial ATP production and biogenesis [2], and that LETM knockdown or overexpression robustly increases or decreases the mitochondrial $\mathrm{Ca}^{2+}$ levels in cells, respectively [3]. Moreover, 
researchers published their findings on the relationship between mitochondrial DNA and cytoplasmic male sterility (CMS) [4-7]. It is believed that the mitochondrial genome is the carrier of CMS factors. In plants, research has shown that a bZIP transcription factor can cause aberrant pollen and low fertility in rice [8]. LETM also has a leucine zipper structure similar to the bZIP transcription factor. So, based on the previous reports and our finding above, it suggests that LETM has a potentially crucial effect and strict expression dose mode in plant stamen development and male fertility. However, so far in plants, the LETM biological function mode remains unknown and largely unexplored.

\section{Materials and Methods}

\subsection{Plant Material}

The studied plant material was upland cotton (Gossypium hirsutum L.), genotype TM-1, which was grown in an experimental field at the South Campus of Shandong Agricultural University in Tai'an, Shandong Province, PR China.

\subsection{Cloning RT-PCR}

Total RNA was isolated from $50 \mathrm{mg}$ plant tissue with a TRI reagent according to the manufacturer's instructions (T9424, Sigma-Aldrich, St. Louis., MO, USA). Then, $0.5 \mu \mathrm{g}$ RNA was used for first-strand cDNA synthesis using the EasyScript ${ }^{\circledR}$ One-Step gDNA Removal and cDNA Synthesis SuperMix (TRAN, Beijing, CN). Next, cDNA was used as a template to amplify full length cDNA of GhLETM1 (Gh_A13G094600) using the following primers: forward 5'-CACCATGGCTTCAAGAGTGATCTTGCG AA-3'; reverse 5'-CTACGATCTTCCTGCTTCAGCAGTG-3' . In addition, the specific unique fragment of GhLETM1 was amplified using the following primers: forward 5'-CACCGTTGTGTGACTGGCT GGATTTATCT-3'; reverse 5'-AATTCCAACTTTCTCCTCCTTTCTG-3'. RT-PCR was performed using an ABI 9902 (Applied Biosystems, Veriti ${ }^{\circledR}$ 96-Well Thermal Cycler 9902), and the PCR program was as follows: $95^{\circ} \mathrm{C}$ for $6 \mathrm{~min}$, followed by 35 cycles of $95^{\circ} \mathrm{C}$ for $30 \mathrm{~s}, 60^{\circ} \mathrm{C}$ for $30 \mathrm{~s}, 72{ }^{\circ} \mathrm{C}$ for $30 \mathrm{~s}$ and finally $72{ }^{\circ} \mathrm{C}$ for $2 \mathrm{~min}$. Then, amplified full length cDNA and the unique fragments were cloned and sequenced for OE (Overexpression) and RNAi (RNA interference) construction, respectively.

\subsection{Vector Construction and Plant Transformation}

To make the OE and RNAi constructs, the full length and the specific, unique cDNA region of GhLETM1 was amplified by RT-PCR as above, and the PCR products were inserted into the plasmid vector pEarlyGate 203 and pB7GWIWG2(II), respectively, using the LR reaction to generate the $P_{35 s}$ :GhLETM1 OE and RNAi constructs [9]. The constructs were respectively transformed into G. hirsutum L. TM-1 mediated by Agrobacterium tumefaciens (LBA4404 strain) according to previous reports [10-12].

\subsection{Molecular Analysis of Transformants}

Total genomic DNA was isolated from young leaves of transgenic plants and WT control using the CTAB method (Murray and Thompson, 1980), which was used for transgenic PCR screening with specific primers (forward 5'-AAGATGGACCCCCACCCACGAG-3'; reverse 5' -AATTCCAA CTTTCTCCTCCTTTCTG-3') that stretched across the pCaMV 35S sequence and GhLETM1 sequence. Total RNA was isolated from plant stamen tissue with TRI reagent according to the manufacturer's instructions (T9424, Sigma-Aldrich, USA), which was used for cDNA synthesis and then for qRT-PCR to assess the quantitative expression level of the native GhLETM1 gene. The qRT-PCR was performed with an Ultra SYBR Mixture (Low ROX) using an Applied Biosystems 7500 Real-Time PCR System (Thermo Fisher, Waltham, MA, USA). The PCR program was as follows: $95^{\circ} \mathrm{C}$ for $10 \mathrm{~min}$, followed by 40 cycles of $95^{\circ} \mathrm{C}$ for $15 \mathrm{~s}$ and $60^{\circ} \mathrm{C}$ for $1 \mathrm{~min}$. The specificity of the amplified PCR products was determined by melting curve analysis $\left(95^{\circ} \mathrm{C}\right.$ for $15 \mathrm{~s}, 60^{\circ} \mathrm{C}$ for $1 \mathrm{~min}, 95^{\circ} \mathrm{C}$ for $15 \mathrm{~s}$ and $60{ }^{\circ} \mathrm{C}$ for 15 s). GhUB7 (DQ116411) of G. hirsutum L. was used as internal control to standardize the results [13]. 
For each gene, the qPCR assays were repeated with triplicate runs. The relative expression levels were measured using the $2^{-\Delta \Delta C t}$ analysis method.

\subsection{Statistical Analysis}

Statistical analyses were performed with one-way ANOVA with a cut-off significance at $p<0.05$ or $p<0.01$ to evaluate the expression differences among the transgenic lines.

\section{Results and Discussion}

In this project, we isolated the homologous GhLETM1 gene in cotton as the model crop for studying stamen development and male fertility because of the big and classic flower organs. Further sequence alignment and comparative analysis of LETM among different plant species based on amino acid sequences are consistent with and extend the report above (Figure 1). GhLETM1 contains the conserved LETM1-like domain (leucine zipper/EF-hand-containing transmembrane protein 1) (Figure 2a), as has been reported in other organisms [1].

With the hypothesis of a strict and sensitive expression dose effect of the LETM gene and corresponding precise control on stamen development, the investigation purpose of the current study is to focus on the ectopic expression dose effect of LETM on plant stamen development and male fertility. So, we constructed GhLETM1 OE and RNAi vectors and performed the transformation in cotton. Positive transgenic ectopic expression lines were identified in OE and RNAi, respectively. Compared to the wild-type (WT) plants, the flowers of the GhLETM1 RNAi and OE transformants showed a size reduction (Figure 2b), with partially shortened filaments (Figure 2c) and partially indehiscent anthers (Figure 2e). Based on the length of the filaments, the phenotypes of the filaments are divided into two categories: shortened filaments and normal filaments. Based on the anther dehiscence status, the phenotypes of the anthers are divided into two categories: anther normal dehiscence and anther indehiscence. We inspected the fertility of the pollen in the OE and RNAi lines. The pollen from the control anthers were completely normal (Figure 2f), while all the pollen of anther indehiscence category presented a yellowish-brown status after staining on the contrary, which indicated that the pollen from the indehiscence anthers are defective (Figure $2 \mathrm{~g}$ ).

The expression level of GhLETM1 in the OE and RNAi lines were further detected by quantitative real-time PCR. GhLETM1 in the D4-13-1, D4-13-18, D5-7-1 and D5-7-14 OE transformants present significantly higher expression compared with the WT plants (Figure 3a). In the RNAi transformants, the gene expression of the D7-4-4, D7-4-13, D7-6-12 and D7-9-5 lines is obviously reduced, but with different levels (Figure 3b).

The degree of abnormality in stamen development was then investigated and analyzed in each transformant above. The D4-13-1 and D4-13-18 OE lines displayed abnormal stamen development with rates of $16 \%$ and $23 \%$, respectively (only the shortened filaments, but all anthers, can normally dehisce). The abnormal stamen rate of the D5-7-1 line was 44\% (within, shortened filaments accounted for $13.6 \%$ and anther indehiscence accounted for $86.4 \%$ ) and $41 \%$ in the D5-7-14 line (14.6\% shortened filaments and $85.4 \%$ anther indehiscence) (Figure 3c). For the RNAi lines, the abnormal stamen rates that appeared in the D7-4-4 lines were 15\% (53.3\% shortened filaments and 46.7\% anther indehiscence), $20 \%$ in the D7-4-13 lines (80.0\% shortened filaments and 20.0\% anther indehiscence); $13 \%$ in the D7-6-12 line (30.8\% shortened filaments and $69.2 \%$ anther indehiscence); and $40 \%$ in the D7-9-5 line $(15.0 \%$ shortened filaments and $85.0 \%$ anther indehiscence) (Figure 3d). 

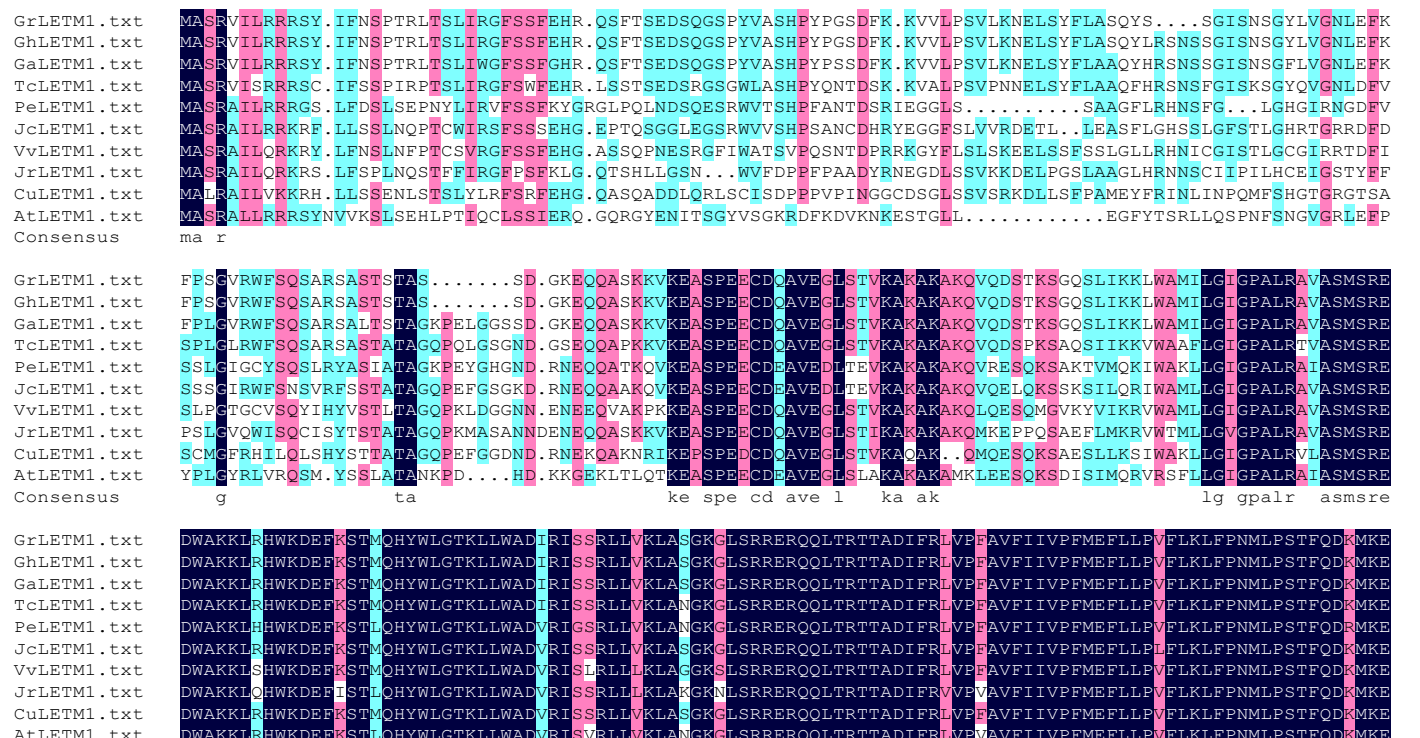

189
193
200
200
189
199
201
199
199
185

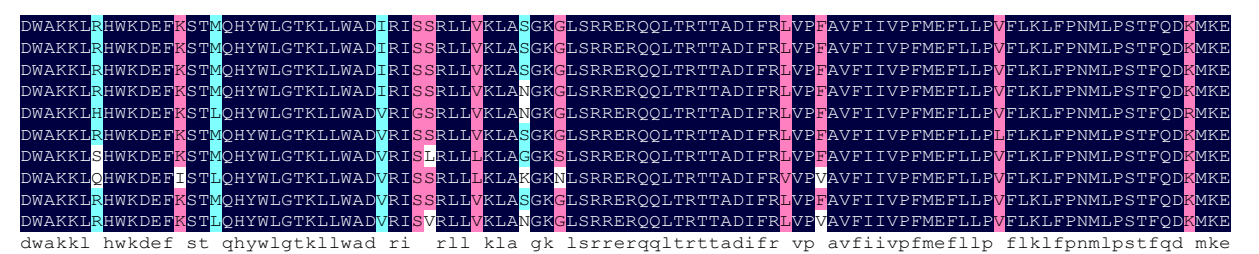

291
295
302
302
291
301
303
301
301
287

GrLETM1.txt

GhLETM1.txt

TCLETM1.txt

PeLETM1. $t x t$

JCLETM1.tX
VVLETM1.tx

JrLETM1. tXt

CuLETM1.txt

Consensus
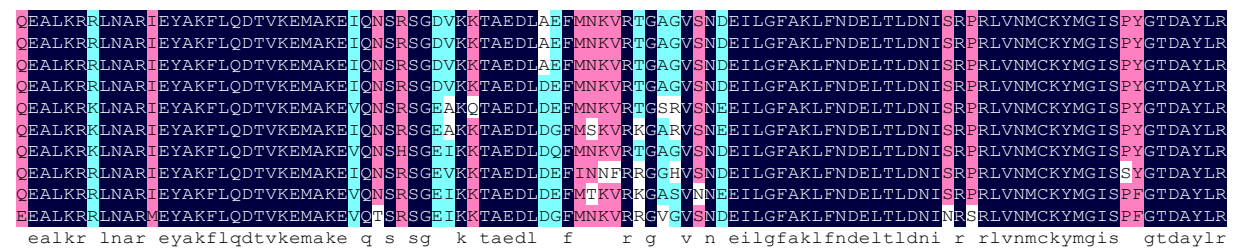

393
397
404
404
393
403
405
403
403
389

GrLETM1. txt

GhLETM1. txt

GaLETM1. txt

PeLETM1.tx

VVLETM1.txt

JrLETM1. tx

CuLETM1. tXt
AtLETM1.txt
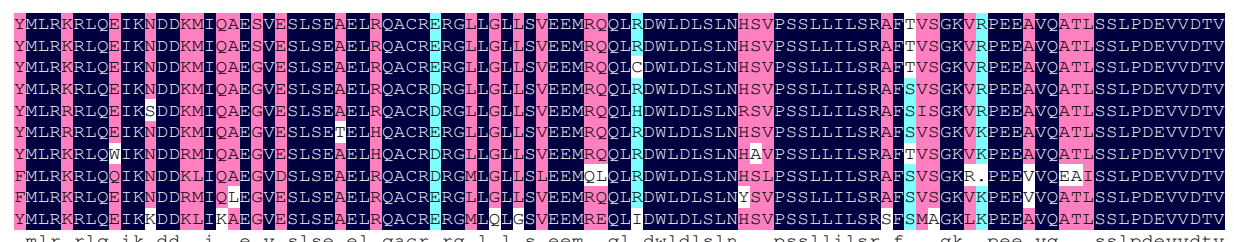

495
499

Consensus

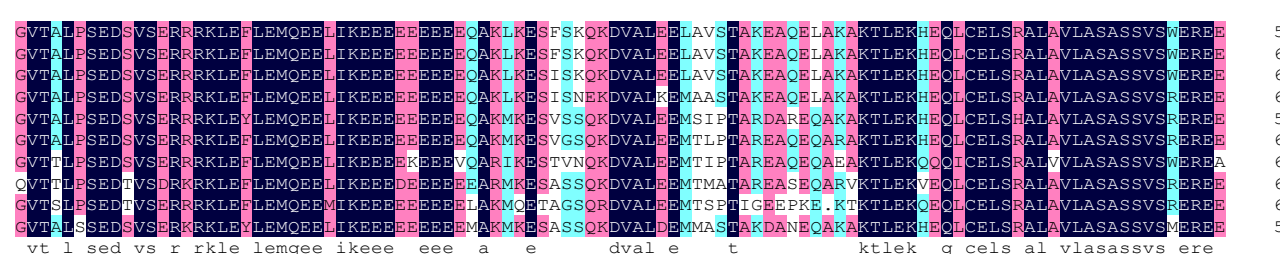

Consensus
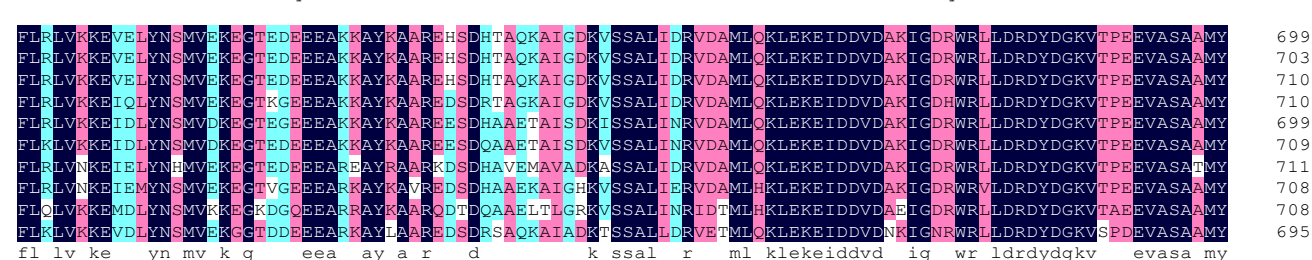

AtLETM1.tx
Consensus

GrLETM1. txt

GhLETM1. $t x$

GaLETM1.tx

TCLETM1. $\mathrm{tx}$
PeLETM1.tx

JCLETM1.txt

VVLETM1. $\mathrm{tx}$

CuLETM1.txt

AtLETM1.txt

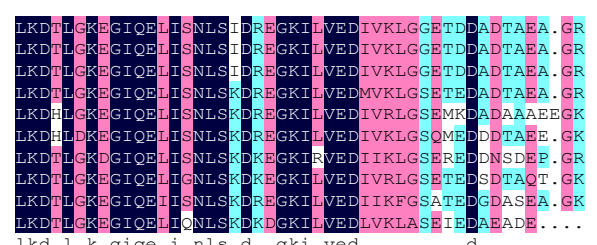

Figure 1. Sequence alignment and comparative analysis of LETM1 among different plants based on amino acid sequences. GhLETM1: LETM1 of Gossypium hisutum; GaLETM1: LETM1of Gossypium arboreum; GrLETM1: LETM1 of Gossypium raimondii; TcLETM1: LETM1 of Theobroma cacao; VvLETM1: LETM1 of Vitis vinifera; JrLETM1: LETM1 of Juglans regia; JCLETM1: LETM1 of Jatropha curcas; PeLETM1: LETM1 of Populus euphratica; CuLETM1: LETM1 of Cucumis; AtLETM1: LETM1 of Arabidopsis thaliana. 


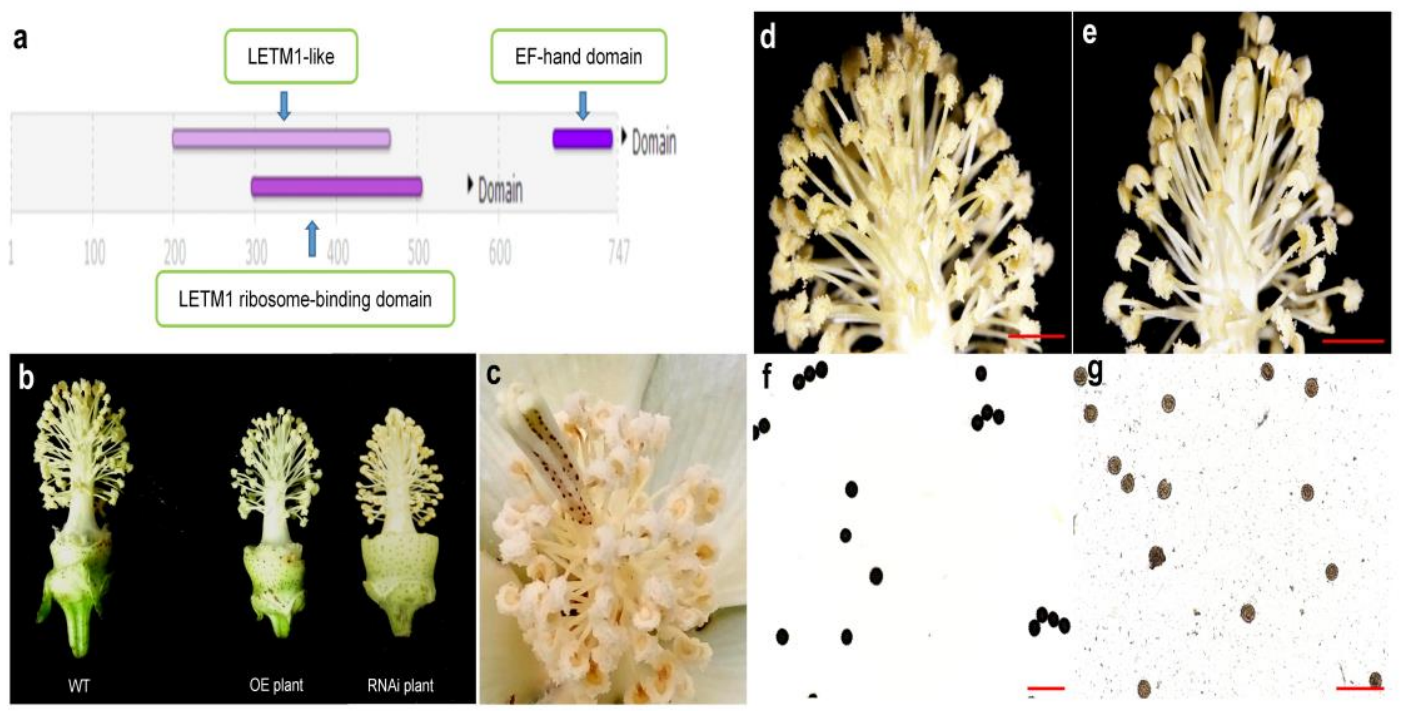

Figure 2. (a) The prediction results of the GhLETM1 protein structure. (b) The flower phenotype of the WT and OE/RNAi transformants. (c) Shortened filaments in the OE and RNAi transformants. (d) Normal dehiscence anthers under a stereomicroscope. Scale bars $=4 \mathrm{~mm}$. (e) Partial indehiscence anthers under a stereomicroscope. Scale bars $=3 \mathrm{~mm}$. (f) Normal pollen activity of the WT plant. (g) Pollen from indehiscence anthers are abortive without activity in the GhLETM1 transformants. Scale bars $=500 \mu \mathrm{m}$.

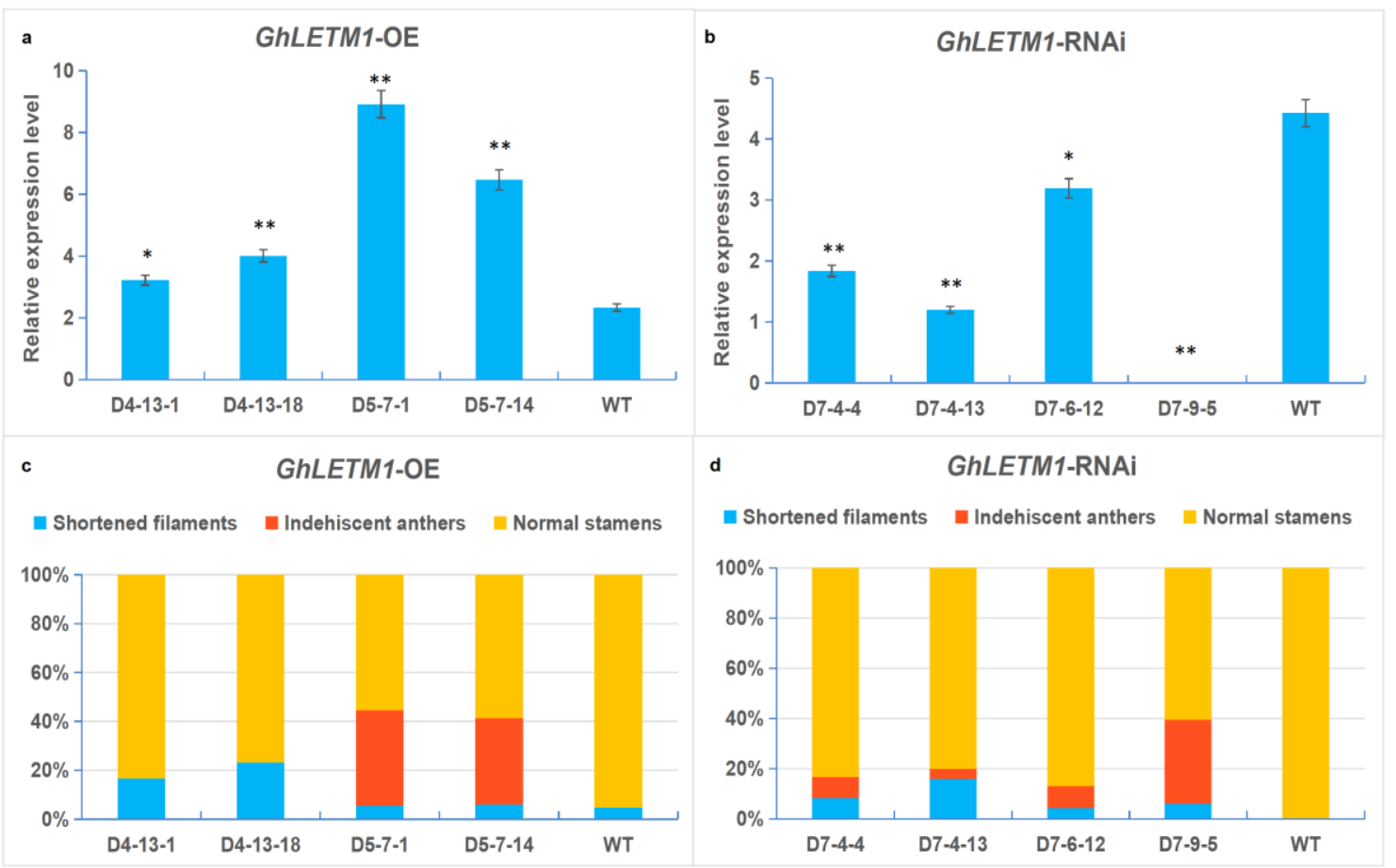

Figure 3. (a,b) The relative expression level analysis of the GhLETM1 gene. Black asterisks indicate statistically significant differences between the WT plant and transgenic plants $\left({ }^{* *} p<0.01 ;{ }^{*} p<0.05\right)$. (a) The relative expression level of the GhLETM1 gene in the OE lines. (b) The relative expression level of the GhLETM1 gene in the RNAi lines. (c,d) Statistics of stamen developmental abnormality in the GhLETM1 transformants. Statistical analyses were performed based on inspection of two thousand filaments with anthers per line. (c) Statistical analysis of abnormal stamen development in the OE lines. (d) Statistical analysis of abnormal stamen development in the RNAi lines. 
Results suggested that, in the OE plants, the higher the GhLETM1 gene, the higher the proportion of abnormal stamens; whereas in the RNAi plants, the lower the GhLETM1 gene, the higher the proportion of abnormal stamens (Figure 3a-d). The above analysis results notably show that both the enhanced and reduced GhLETM1 expression level were significantly correlated with degree of abnormality in stamen development. These investigations revealed that GhLETM1 expression should be maintained at a certain level with a dose effect. Excessive and deficient expression doses would lead to abnormal stamens with shortened filaments and abortive anthers. At the same time, the results also showed that, in the lines which presented the severely defective stamens, phenotype greatly disturbed expression of GhLETM1 (like in OE lines D4-13-1, D4-13-18, D5-7-1 and D5-7-14; RNAi lines D7-4-13 and D7-9-5). The higher proportion of shortened filaments is along with the opposite lower ratio of anther abortion and vice versa. The results indicated that the shortened filament phenotype was negatively correlated with anther abortion. It suggested that the two molecular pathways of GhLETM1 regulation on filament shortening and anther abortion are interdependent (Figure 3c,d).

While we detected the distinctly affected phenotypes of the stamen, we also inspected the other main organs and did not find obvious abnormal phenotypes, i.e., visible to the naked eye. There likely occurred internal trait variation and in vivo physiological characteristic changes in the ectopic expressed lines. Although our results indicate that the gene has a distinct effect on stamen development, we cannot conclude that only this developmental process is ectopically affected.

The mitochondrial transmembrane conserved domain contained in the GhLETM1 sequence implies that GhLETM1 can play roles in plant stamen development and male fertility maintenance, likely as the unit of mitochondrial respiratory chain complexes, especially involved in respiratory chain biotransformation. However, future research is necessary to explain how the precise regulation is established and to elucidate the molecular pathway and mechanisms regulating stamen development and male fertility in plants.

A multigene family with gene duplication is a common and abundant event in plants; there are at least six LETM members that exist in the cotton (Gossypium hirsutum L.) genome. Still, all LETM gene members' functions and relationships are poorly understood so far. The findings in our current study are consistent with and extend the gene balance hypothesis, which suggests that the expression levels of family gene members encoding the components of crucial molecular pathways need to be strictly maintained for precise and proper function [14]. At the same time, further experiments will be necessary for understanding the gene members' interaction and molecular regulation network underlying stamen development and male fertility during this concerted important process.

In this study, ectopic expressions of the GhLETM1 gene reveal a sensitive dose effect and corresponding precise control on concerted development of stamen and male fertility in cotton. This study presents significant findings on mining new genes related to male sterility in plants, which are of fundamental and practical importance in crop science, holding significant promise for its advancement in gene engineering on new male sterile lines to cultivate hybrid varieties in crop breeding. For example, we can edit gene promoters through CRISPR technology that can regulate the level of gene expression to convert the fertility of plants. In addition, we can further investigate the external optimized induction conditions for the gene expression and create environmentally induced male sterile lines. Thus, the efficiency in crop hybrid breeding can be greatly promoted using new types of male sterile lines by artificial regulation.

\section{Conclusions}

The functional mode of the potentially crucial LETM gene involved in the developmental process of stamen development and male fertility remains unknown and largely unexplored in plants. Based on the above ectopic expression analysis, both excessive and deficient expression of the GhLETM1 gene lead to defective stamen development, resulting in partially shortened filaments and indehiscent anthers with pollen abortion. Further inspection also revealed that there is a sensitive ectopic expression dose effect of the GhLETM1 gene on filament elongation and anther dehiscence in cotton. It suggests 
that the gene could be involved in crucial molecular pathways and need to be strictly maintained for its proper function for concerted stamen development and male fertility. The investigation results also showed that the shortened filament phenotype was negatively correlated with anther defects in the seesaw model under the ectopic expression of the GhLETM1 gene.

Author Contributions: Data curation, Y.F., H.G. (Haixia Guo), H.G. (Huihui Guo), X.L., Z.G. and C.Z.; Formal analysis, L.Z., Y.Z. (Yao Zhang), Y.F. and F.Z.; Funding acquisition, H.W.; Investigation, L.Z., Y.Z. (Yao Zhang) and Y.F.; Methodology, Y.F., H.G. (Haixia Guo), H.G. (Huihui Guo), J.W., Y.Z. (Yunlei Zhao), X.L., Y.S. and C.C.; Project administration, H.G. (Huihui Guo), J.W. and Y.S.; Resources, J.W., H.W., Y.Z. (Yunlei Zhao), X.L., Z.G., C.Z. and C.C.; Supervision, F.Z.; Writing—original draft, L.Z. and Y.Z. (Yao Zhang); Writing—review and editing, L.Z., H.G. (Haixia Guo), C.C. and F.Z. All authors have read and agreed to the published version of the manuscript.

Funding: This work was supported by the National Key Research and Development Program (2016YFD0100306, 2016YFD0100303), NSFC (31401428) and Taishan Scholar Talent Project from China (TSQN20161018). The funding bodies had no role in the design of the study and collection, analysis, and interpretation of data and in writing the manuscript.

Acknowledgments: We are grateful to Chengzhi Jiao and Xinpeng Gao (Shandong Agricultural University) for field management.

Conflicts of Interest: The authors declare no conflict of interest.

\author{
Abbreviations \\ Gh Gossypium hirsutum L. \\ LETM Leucine zipper/EF-hand-containing transmembrane protein \\ OE Overexpression \\ RNAi RNA interference \\ CMS Cytoplasmic Male Sterility \\ qPCR Quantitative Polymerase Chain Reaction
}

\title{
References
}

1. Schlickum, S.; Moghekar, A.; Simpson, J.C.; Steglich, C.; O’Brien, R.J.; Winterpacht, A.; Endele, S.U. LETM1, a gene deleted in Wolf-Hirschhorn syndrome, encodes an evolutionarily conserved mitochondrial protein. Genomics 2004, 83, 254-261. [CrossRef]

2. Piao, L.; Li, Y.; Kim, S.J.; Byun, H.S.; Huang, S.M.; Hwang, S.K.; Yang, K.J.; Park, K.A.; Won, M.; Hong, J.; et al. Association of LETM1 and MRPL36 contributes to the regulation of mitochondrial ATP production and necrotic cell death. Cancer Res. 2009, 69, 3397-3404. [CrossRef]

3. Jiang, D.; Zhao, L.; Clapham, D.E. Genome-wide RNAi screen identifies Letm1 as a mitochondrial Ca $2_{+} / \mathrm{H}_{+}$ antiporter. Science 2009, 326, 144-147. [CrossRef]

4. Levings, C.S.; Pring, D.R. Restriction endonuclease analysis of mitochondrial DNA from normal and Texas cytoplasmic male-sterile maize. Science 1976, 193, 158-160. [CrossRef] [PubMed]

5. Ducos, E.; Touzet, P.; Boutry, M. The male sterile G cytoplasm of wild beet displays modified mitochondrial respiratory complexes. Plant J. 2001, 26, 171-180. [CrossRef] [PubMed]

6. Luo, D.; Xu, H.; Liu, Z.; Guo, J.; Li, H.; Chen, L.; Fang, C.; Zhang, Q.; Bai, M.; Yao, N.; et al. A detrimental mitochondrial-nuclear interaction causes cytoplasmic male sterility in rice. Nat. Genet. 2013, 45, 573-577. [CrossRef] [PubMed]

7. Touzet, P.; Meyer, E.H. Cytoplasmic male sterility and mitochondrial metabolism in plants. Mitochondrion 2014, 16, 166-171. [CrossRef] [PubMed]

8. Zou, M.J.; Guan, Y.C.; Ren, H.B.; Zhang, F.; Chen, F. A bZIP transcription factor, OsABI5, is involved in rice fertility and stress tolerance. Plant Mol. Biol. 2008, 66, 675-683. [CrossRef] [PubMed]

9. Helliwell, C.A.; Wesley, S.V.; Wielopolska, A.J.; Waterhouse, P.M. High-throughput vectors for efficient gene silencing in plants. Funct. Plant Biol. 2002, 29, 1217-1225. [CrossRef]

10. Liu, Z.; Zhu, Z.; Zhang, T. Development of Transgenic CryIA(c) + GNA Cotton Plants via Pollen Tube Pathway Method Confers Resistance to Helicoverpa Armigera and Aphis Gossypii Glover. Methods Mol. Biol. 2013, 958, 199-210. [PubMed] 
11. Bibi, N.; Fan, K.; Yuan, S.; Ni, M.; Ahmed, I.M.; Malik, W.; Wang, X. An efficient and highly reproducible approach for the selection of upland transgenic cotton produced by pollen tube pathway method. Aust. J. Crop Sci. 2013, 7, 1714-1722.

12. Wang, M.; Zhang, B.; Wang, Q. Cotton Transformation via Pollen Tube Pathway. Methods Mol. Biol. 2013, 958, 71-77. [PubMed]

13. Guo, K.; Du, X.; Tu, L.; Tang, W.; Wang, P.; Wang, M.; Liu, Z.; Zhang, X.J. Fibre elongation requires normal redox homeostasis modulated by cytosolic ascorbate peroxidase in cotton (Gossypium hirsutum). Exp. Bot. 2016, 67, 3289-3301. [CrossRef] [PubMed]

14. Birchler, J.A.; Veitia, R.A. Gene Balance Hypothesis: Connecting Issues of Dosage Sensitivity across Biological Disciplines. Proc. Natl. Acad. Sci. USA 2012, 109, 14746-14753. [CrossRef] [PubMed]

(C) 2020 by the authors. Licensee MDPI, Basel, Switzerland. This article is an open access article distributed under the terms and conditions of the Creative Commons Attribution (CC BY) license (http://creativecommons.org/licenses/by/4.0/). 